\title{
Dysphagia From Extrinsic Compression of Esophagus by Pericardial Effusion
}

\author{
Hemender Singh Vats, MD
}

A

96-year-old woman was admitted for evaluation of progressive dysphagia and generalized weakness of 1-year duration. Dysphagia, although initially only to solid foods, had progressed to foods of all consistencies and even plain water ingestion induced vomiting. Past medical history was significant for gastroesophageal reflux disease and iron deficiency anemia. The only medication she was taking was omeprazole. Examination revealed a frail, dehydrated woman with a blood pressure of $91 / 56 \mathrm{~mm} \mathrm{Hg}$. Other vitals were stable. Heart sounds were distant and the rest of the examination was unremarkable.

Laboratory tests revealed anemia consistent with iron deficiency. She was hypoalbuminemic $(2.9 \mathrm{~g} / \mathrm{dL})$. The remaining results were largely normal. Chest x-ray revealed cardiomegaly. Fluoroscopic swallow evaluation showed a normal oropharyngeal response but markedly delayed esophageal clearance suggesting normal swallowing but retention of food inside the esophagus. A barium swallow revealed a smooth, almost complete obstruction of the esophagus below the level of the carina suggestive of extrinsic compression (figure 1A). An esophagogastrodudenoscopy was normal and confirmed mild extrinsic compression in the lower esophagus. A two-dimensional echocardiogram confirmed a large pericardial effusion. Computed tomogram of the chest confirmed the pericardial effusion but did not reveal any other mediastinal or pulmonary pathology (figure 1B).

The options for treatment included therapeutic drainage of the pericardial effusion to relieve the pressure on the esophagus and correction of iron deficiency. These were presented to the patient. However, the patient

Figure 1. (A) Barium esophagogram with arrows pointing at the smooth filling defect in esophagus suggestive of extrinsic compression. (B) Computed tomographic scan image with arrows pointing to large pericardial effusion.
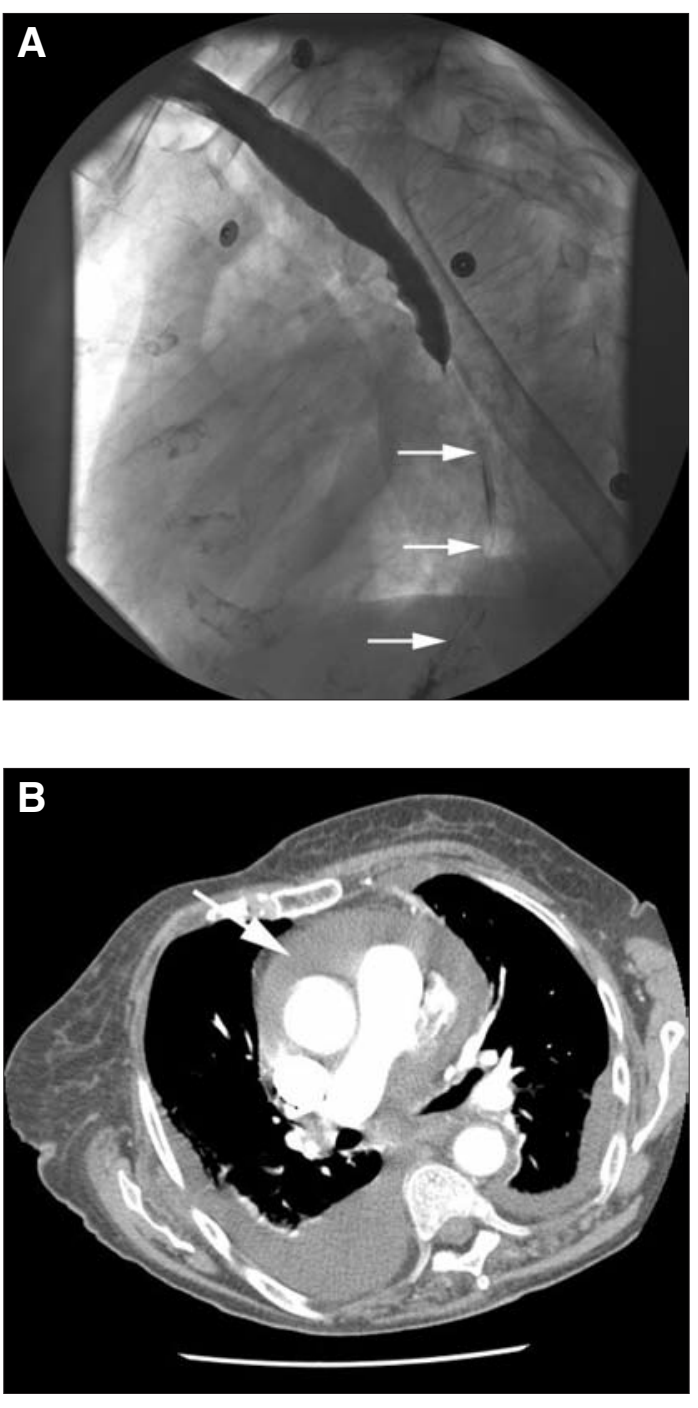

Reprint Requests:

Hemender Singh Vats, MD

Department of Internal Medicine

Marshfield Clinic

1000 North Oak Avenue

Marshfield, Wisconsin 54449, USA

Tel: 7I 5-387-5537

Fax: 7I5-389-3808

Email: vats.hemender@marshfieldclinic.org.
Received: August 17,2007

Revised: November 27, 2007

Accepted: December 19, 2007

doi: $10.3|2| / \mathrm{cmr} .2008 .780$

The Aperture, like the opening in the lens of a microscope that allows light to pass through, is a forum for art, humor, and images that provides a portal for new or different views of medicine and research. 
adamantly refused any further workup or treatment, including any means of artificial feeding. She was subsequently transferred to palliative care service where she died the following week.

\section{Discussion}

Dysphagia is a common symptom in many elderly patients. Although true prevalence of dysphagia is unknown, epidemiological studies estimate a prevalence rate of $16 \%$ to $22 \%$ among individuals over 50 years of age. ${ }^{1}$ The two basic mechanisms responsible for dysphagia include either disordered motor function or mechanical obstruction of the oropharynx or esophagus. Oropharyngeal dysphagia (also known as transfer dysphagia) refers to abnormalities in transfer of food from the mouth to oropharynx and upper esophagus. The common causes of oropharyngeal dysphagia include neurological deficits and neuromuscular disorders. Esophageal dysphagia is caused by motor disorders (achalasia, motility disorders, and calcinosis, Raynaud phenomenon, esophageal dysmotility, sclerodactyly, telangiectasia [CREST] syndrome) or mechanical lesions causing obstruction. These lesions are usually intrinsic (strictures, rings, webs and tumors). Rarely extrinsic esophageal compression (mediastinal masses, vascular compression and surgical changes) is the underlying cause of dysphagia. Compression of the esophagus by an enlarged left atrium (e.g., from mitral stenosis) has been documented, however, compression by a pericardial effusion has not been reported. 1,2

A detailed history is essential to identify the cause of the dysphagia. Subsequent workup is directed by the initial assessment. The cornerstone of the oropharyngeal dysphagia diagnosis is the fluoroscopic swallowing evaluation supplemented by barium swallow radiography, video laryngoscopy, upper esophageal endoscopy and esophageal manometry. Esophageal dysphagia is initially worked up with barium studies, although in some cases, esophagogastric endoscopy is done directly. If no intraluminal cause is demonstrated, attention is directed to motility disorders and extrinsic compression, which can be evaluated with manometery studies and imaging studies. ${ }^{3}$

Treatment for dysphagia is directed at the identified cause. Outcome from treatment is better when the mechanical obstruction can be removed surgically or relieved by other measures (ablation, dilatation, radiation, stenting, etc). However, surgically correctible causes cannot be identified in most patients with oropharyngeal dysphagia. The goal of treatment in these patients is to ensure effective transfer of food to upper esophagus without aspiration which is achieved by postural, nutritional and behavioral modifications (collectively termed as swallowing rehabilitation). ${ }^{4}$

This case presents pericardial effusion as a cause of extrinsic compression of the esophagus. It is possible that the prolonged bedridden and debilitated state of the patient aggravated the mechanical pressure exerted by the large pericardial effusion on the lower half of the esophagus. Therapeutic drainage of the effusion would have potentially relieved the patient's symptoms.

\section{References}

1. Lind CD. Dysphagia: evaluation and treatment. Gastroenterol Clin North Am 2003;32:553-575.

2. Achem SR, Devault KR. Dysphagia in aging. J Clin Gastroenterol 2005;39:357-371.

3. Richter JE. Practical approach to the diagnosis and treatment of esophageal dysphagia. Compr Ther 1998;24:446-453.

4. Cook IJ, Kahrilas PJ. AGA technical review on management of oropharyngeal dysphagia. Gastroenterology 1999; 116:455-478.

\section{Author Affiliation}

Hemender Singh Vats, MD

Department of Internal Medicine

Marshfield Clinic

Marshfield, Wisconsin, USA 